\title{
Erratum to: Solution behavior of aqueous mixtures of low and high molecular weight hydrophobic amphiphiles
}

\author{
Carlos Rodríguez-Abreu • Margarita Sanchez-Domínguez • Bojan Šarac • \\ Marija Bešter Rogač • Rekha Goswami Shrestha • Lok Kumar Shrestha • \\ Dharmesh Varade • Goutam Ghosh • Vinod K. Aswal
}

Published online: 8 May 2010

(C) Springer-Verlag 2010

\section{Erratum to: Colloid Polym Sci DOI 10.1007/s00396-010-2188-8}

The original version of this article unfortunately contained mistakes. Several author corrections in different places were not carried-out due to technical problems. The correct version of the article is shown below.

\begin{abstract}
Mixtures of a hydrophobic triblock copolymer (L121, $\mathrm{PEO}_{5} \mathrm{PPO}_{68} \mathrm{PEO}_{5}$ ) and a hydrophobic anionic surfactant (AOT, Sodium bis(2-ethylhexyl)sulfosuccinate), each alone forming turbid vesicular solutions in water, aggregate to produce a thermodynamically stable, transparent and isotropic solution. Mixed AOT/L121 aggregates
\end{abstract}

The online version of the original article can be found at http://dx.doi. org/10.1007/s00396-010-2188-8.

Electronic supplementary material The online version of this article (doi:10.1007/s00396-010-2213-y) contains supplementary material, which is available to authorized users.

C. Rodríguez-Abreu ( $\bowtie)$

Instituto de Química Avanzada de Cataluña,

Consejo Superior de Investigaciones Científicas (IQAC-CSIC),

Jordi Girona 18-26,

08034 Barcelona, Spain

e-mail: craqci@iiqab.csic.es

C. Rodríguez-Abreu $\cdot$ M. Sanchez-Domínguez

CIBER de Bioingeniería,

Biomateriales y Nanomedicina (CIBER-BBN),

Barcelona, Spain

B. Šarac $\cdot$ M. B. Rogač

Faculty of Chemistry and Chemical Technology,

University of Ljubljana,

Aškerčeva 5,

S1-1000 Ljubljana, Slovenia could be confirmed by fluorescence, surface tension, differential scanning calorimetry (DSC) and isothermal titration calorimetry (ITC). In an isotropic region, where mixed aggregates are formed, there is a synergistic interaction between monomers of AOT and L121 in the mixture. In addition, Small Angle Neutron Scattering (SANS) experiments provided evidence that mixed aggregates have the shape of either spheres (with a certain polydispersity) or very short ellipsoids (axial ratio below 2), confirming a transition from giant multilamellar vesicles to small aggregates upon mixing the two hydrophobic amphiphiles. Upon dilution, the morphology changes to disk-like. From an examination of the results of all the methods the peculiar behavior of the mixed AOT/L121 system is explained.

R. G. Shrestha • L. K. Shrestha • D. Varade

Graduate School of Environment and Information Sciences,

Yokohama National University,

Tokiwadai 79-7, Hodogaya-ku,

Yokohama 240-8501, Japan

G. Ghosh

UGC-DAE Consortium for Scientific Research, B.A.R.C.,

Trombay,

Mumbai 400 085, India

V. K. Aswal

Solid State Physics Division, B.A.R.C., Trombay,

Mumbai 400 085, India 
Keywords SANS · DSC $\cdot$ ITC · Dynamic surface tension . Block copolymers $\cdot$ AOT $\cdot$ Micelles

\section{Introduction}

It is known that mixing surfactants usually enhances the performance of products. Mixing can be also unavoidable as a result of fabrication processes. Therefore, it is important to understand the features of mixed systems, particularly in terms of their phase behavior and structure.

Poly(ethylene oxide)-Poly (propylene oxide) (PEO-PPO) amphiphilic block copolymers are versatile compounds whose hydrophile-hydrophobe balance can be tailored by changing the PEO (hydrophile) or PPO (hydrophobe) chain lengths or their molecular weights [1-4]. The change in the chain conformation and solvation with temperature or by addition of selective solvents also allows surface curvature changes that give rise to a very rich phase behavior and a variety of self-organizing structures; this subject has been studied extensively. Block copolymer nanostructures are often used in conjunction with ionic surfactants to modulate their functional properties [5-11]. Due to synergistic interaction with these surfactants, their performance improves as compared to that of individual components [12-14] in applications such as emulsification [15] and stabilization of dispersions [16]. Since the functionality of copolymer assemblies critically depends on the structure and the properties of mixed assemblies, clear understanding of the basic aggregation mechanism of block copolymer assemblies with different secondary species, such as ionic surfactants, proteins, etc., is crucial. This is also true for advanced applications such as the preparation of nanomaterials [17], in which mixing surfactants allows to tune the shape and curvature of the aggregates used as templates in order to obtain the desired structures.

In a previous report, for the mixtures of a hydrophobic triblock copolymer (L121, $\left.\mathrm{PEO}_{5} \mathrm{PPO}_{68} \mathrm{PEO}_{5}\right)$ and a hydrophobic anionic surfactant (AOT, Sodium bis(2-ethylhexyl) sulfosuccinate), we found complete mixing of the two surfactants to form mixed aggregates. A phase diagram of this system is shown in Fig. 1 [18]. At both low and high $\mathrm{AOT} / \mathrm{L} 121$ ratios, two liquid phases are present and samples look turbid, due to the presence of large vesicles. At intermediate AOT/L121 ratios, the solutions are singlephase, transparent and isotropic. Still, some questions remained opened concerning the main causes of this unexpected behavior.

Both micelle-to-vesicle and vesicle-to-micelle transitions are relevant for model biological membranes and delivery systems [19-21] in which the presence of PEO chains can provide enhanced circulation time [22]. Such transition has been reported previously in polymer-surfactant systems, but mainly for mixtures where either the polymer or the surfactant is hydrophilic [23, 24]. In the present case both surfactant and polymer are hydrophobic, which imparts particular features to the system. To get more insight on the structure of the mixed L121+AOT aqueous solutions, we have further studied this system using small angle neutron scattering (SANS), tensiometry, fluorescence and differential scanning calorimetry (DSC) techniques.

\section{Experimental}

\section{Materials}

Sodium bis(2-ethylhexyl)sulfosuccinate (AOT) was purchased from Sigma-Aldrich. The poly(ethylene oxide)poly(propylene oxide)-poly(ethylene oxide) (PEO-PPOPEO) triblock copolymer Pluronic L121 was a gift from BASF Corp. The same batch of L121 was used in all experiments. The copolymer molecular formula can be represented as $\mathrm{PEO}_{5} \mathrm{PPO}_{68} \mathrm{PEO}_{5}$. Pyrene $(98 \%)$ was purchased from Fluka. All substances were used without further purification. Millipore-Q treated water was used for sample preparations except for SANS were $\mathrm{D}_{2} \mathrm{O}$ was used.

\section{Methods}

\section{Phase diagrams}

Proper amounts of components were weighed in ampoules that afterwards were flame sealed, homogenised and kept in a water bath at several temperatures for equilibration. Phases were identified by visual observation through

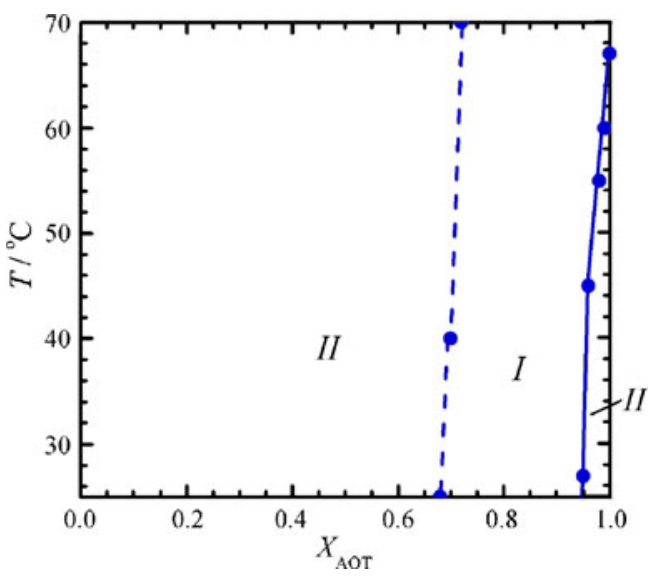

Fig. 1 Phase behavior of AOT/L121/water systems as a function of AOT bulk mol fraction $\left(X_{\mathrm{AOT}}\right)$ in the AOT+L121 mixture (solventfree basis). The total amphiphile (AOT + L121) concentration is kept constant at $5 \mathrm{wt} \%$. I and II indicate one- and two-phase regions, respectively. Adapted from Ref. [18] 
crossed polarisers, polarized optical microscopy (Leica Reichert Polyvar 2 instrument) and Small Angle X-Ray Scattering.

\section{Small Angle X-ray Scattering (SAXS)}

SAXS measurements were performed using a S3 MICRO instrument (Hecus X-ray Systems, from Graz, Austria) equipped with a GENIX microfocus X-ray source and a FOX 2D point-focusing element (both from Xenocs, Grenoble). The $d$ spacings of liquid crystalline phases was determined by the Bragg equation, $d=2 \pi / q$, where $q$ is the scattering vector.

Small-Angle Neutron Scattering (SANS) SANS experiments were carried out in the instrument installed in the Guide Tube (GT) laboratory, Dhruva reactor hall, BARC, Trombay, India. Details about the SANS instruments and analysis procedure can be found elsewhere $[25,26]$. The $Q$ range of the diffractometer is $0.18-3 \mathrm{~nm}^{-1}$. Hydrogenated surfactant aggregates were contrasted against $\mathrm{D}_{2} \mathrm{O}$. Experiments were carried out at $25^{\circ} \mathrm{C}$, and samples were loaded in $5 \mathrm{~mm}$ path-length rectangular quartz cells. To improve the statistics on the SANS patterns each measurement was taken for 8-10 hours.

In a SANS experiment one measures the differential scattering cross section $\left(d \sum(Q) / d \Omega\right)$ per unit volume. For a system of monodisperse particles, it is given by [27]

$$
\frac{d \sum(Q)}{d \Omega}=n\left(\rho_{p}-\rho_{s}\right)^{2} V^{2} P(Q) S(Q)+B
$$

where $n$ is the number density of the particles, $\rho_{p}$ and $\rho_{s}$ are, respectively, the scattering length densities of the particle and the solvent, and $V$ is the volume of the particle. $P(Q)$ is the intra-particle form (shape) factor and $S(Q)$ is the inter-particle structure factor. $B$ is a constant term that represents the incoherent scattering background, which is mainly due to hydrogen in the sample.

The SASFIT program (developed at Paul Scherrer Institute, Laboratory for Neutron Scattering, Villigen, Switzerland) was used for data fitting. For a $5 \mathrm{wt} \%$ total (AOT+L121) surfactant concentration, the $P(Q)$ contribution was tested for ellipsoidal aggregates as well as spherical aggregates and $S(Q)$ has been calculated using the model of Hayter and Penfold [28] which assumes screened Coulomb interactions between the charged aggregates. The micellar dimensions, aggregation number and fractional charge have been determined from the analysis. For the ellipsoidal aggregates, the semimajor axis $(a)$, the semiminor axis $(b)$, the fractional charge $(\alpha=z / N$, where $z$ is the micellar charge in $e^{-}$and $N$ is te aggregation number) and the hard sphere radius are the fitting parameters in analysing the SANS data. For the spherical aggregates, the sphere radius $(r)$, the polydispersity, the fractional charge and the hard sphere radius are the fitting parameters. For the ellipsoidal aggregates, the aggregation number is calculated by the relation $N=4 \pi a b^{2} /\left(3 \overline{\mathrm{V}_{\mathrm{m}}}\right)$, whereas for the spherical aggregates it is calculated by the relation $N=4 \pi r^{3} /\left(3 \overline{\mathrm{V}_{\mathrm{m}}}\right)$. In both cases $\overline{\mathrm{V}_{\mathrm{m}}}$ is the average surfactant monomer volume. The latter was calculated for each sample as an average surfactant monomer volume taking into account the corresponding contributions from AOT and L121, $\overline{V_{\mathrm{m}}}=V_{\mathrm{mAOT}} \cdot X_{\mathrm{AOT}}+V_{\mathrm{mL} 121} \cdot$ $X_{\mathrm{L} 121}$, where $\mathrm{X}_{\mathrm{AOT}}$ and $\mathrm{X}_{\mathrm{L} 121}$ are the mole fractions of AOT and L121, respectively, in a water-free basis. For AOT, the molecular volume $\mathrm{V}_{\text {mAOT }}$ used was $650 \AA^{3}$ [29] and for L121, $\mathrm{V}_{\mathrm{mL} 121}$ was $7100 \AA^{3}$ [30]. In addition, a small contribution to the form factor was added taking into account the Gaussian chains from L121 for all the samples of the first series.

For a $0.5 \mathrm{wt} \%$ surfactant concentration, the form factor $P$ $(Q)$ for a disk with radius $R$ and height $h$ was used. Neither polydispersity nor $S(Q)$ were considered, as the low concentration allowed to neglect the effect of interactions on the scattering intensity.

Steady State Fluorescence Steady state fluorescence of pyrene in water was measured using a Varian Cary Eclipse spectrometer. Pyrene was excited at a wavelength of $335 \mathrm{~nm}$ and the emission intensity from 350 to $500 \mathrm{~nm}$ was recorded. The excitation and emission slit openings were set at 5 and $2.5 \mathrm{~nm}$, respectively. The scan speed was set at $600 \mathrm{~nm} / \mathrm{min}$. All spectra were averaged over two scans.

Equilibrium and dynamic surface tension The equilibrium surface tension of aqueous mixed surfactant solution was measured at $25^{\circ} \mathrm{C}$ by the Wilhelmy plate technique (K100 tensiometer, Krüss, Germany). Dynamic surface tension was measured by the maximum bubble-pressure method using a bubble pressure tensiometer BP2 (Krüss, Germany) at $25 \mathrm{C}$. Surface ages of bubbles were measured between $5 \mathrm{~ms}$ to $50 \mathrm{~s}$.

Differential Scanning Calorimetry (DSC) DSC traces were recorded with a Setaram Micro DSC III. The samples $(\sim 0.5 \mathrm{~g})$ were introduced in Hastelloy pans and heated at a rate of $1.5^{\circ} \mathrm{C} / \mathrm{min}$ under nitrogen atmosphere. A pan filled with Millipore water was used as a reference.

Isothermal Titration Calorimetry (ITC) ITC experiments were performed by a MicroCal ITC microcalorimeter. The syringe of calorimeter $(\mathrm{V} \sim 0.3 \mathrm{~mL})$ was filled with aqueous solutions of AOT or a mixture of AOT and L121 which 
were then titrated into a cell containing $1.384 \mathrm{~mL}$ of water. Each experiment, performed at $25^{\circ} \mathrm{C}$, consisted of 99 subsequent additions $(\mathrm{V}=3 \mu \mathrm{L})$ of titrant solution.

\section{Results and discussion}

Phase diagram

The partial ternary phase diagrams of L121-AOT-water systems are presented in Fig. 2. Single AOT and single L121 aqueous systems form lamellar liquid crystals in water. At $25^{\circ} \mathrm{C}$, a lamellar phase is found from ca. $15 \mathrm{wt} \%$ of AOT in water whereas the same liquid crystal is present from ca. $60 \mathrm{wt} \% \mathrm{~L} 121$ in water. Note that minute addition of AOT greatly stabilizes liquid crystal phases in the binary L121-water system; therefore, the liquid crystal region looks greatly expanded. Interestingly, mixing of the lamellar phases at certain AOT/L121 ratio results in single isotropic regions. In the dilute region, such an isotropic region is found between two multiphase regions in which vesicles or lamellar dispersions can be formed. The SAXS spectra for the lamellar liquid crystals at low AOT content showed three peaks at position ratios 1:2:3 corresponding to lamellar structures. On the other hand, the SAXS patterns for liquid crystal samples at high AOT content showed two well resolved peaks at position ratios 1:2 and one broad peak with a maximum at higher $q$ values. In AOT aqueous systems, SAXS spectra also feature such a broad peak, which was attributed to the form factor of the defective AOT lamellar phase [31].

\section{Fluorescence measurements}

The pyrene emission dependence of the solvent polarity is expressed in terms of the ratio $I_{1} / I_{3}$ of the intensities of the first and the third vibronic bands. This ratio decreases with increasing hydrophobicity. Pyrene, having low water solubility, is expected to partition preferentially to the most hydrophobic regions of a self organized system in solution.

A simple mixing relationship [32] for the ratio of vibronic band intensities in the present binary mixed amphiphilic system would be

$$
\left(I_{1} / I_{3}\right)_{\mathrm{M}}=X_{\mathrm{AOT}}\left(I_{1} / I_{3}\right)_{\mathrm{AOT}}+\left(1-X_{\mathrm{AOT}}\right)\left(I_{1} / I_{3}\right)_{\mathrm{L} 121}
$$

where $\left(I_{1} / I_{3}\right)_{\mathrm{M}},\left(I_{1} / I_{3}\right)_{\mathrm{AOT}},\left(I_{1} / I_{3}\right)_{\mathrm{L} 121}$ are the ratios of intensities of the mixture, AOT micellar solutions, and L121 aqueous solutions above the aggregation concentration, respectively, and $X_{\mathrm{AOT}}$ is the mole fraction of AOT in the surfactant mixture. It is worth mentioning that in the present study the obtained experimental value for $\left(I_{1} / I_{3}\right)_{\mathrm{L} 121}$ is close to those reported for other pluronics with a similar
PPO chain length [33], but much higher than that of pure PPO, indicating that PPO chains in the aggregate could be somewhat hydrated.

As can be seen in Fig. 3, the actual values for $\left(I_{1} / I_{3}\right)_{\mathrm{M}}$ are much higher than those given by Eq. 2 within a wide range of AOT mol fractions, which indicates that the contribution of L121 to the mixture is also higher than predicted. It suggests that the fluorescent probe is mostly surrounded by L121 lipophilic PPO chains in the micellar core. The $I_{1} / I_{3}$ ratio does not decrease significantly up to $X_{A O T} \approx 0.7$. After this value, which is close to the lower concentration boundary of the single phase region (see Fig. 1), there is sharp drop in the $I_{1} / I_{3}$ ratio. In some cases, vesicles or layers are less hydrated than aggregates and therefore such a drop would not be expected. There might be two reasons for such a behavior. One could be that the mol fraction of AOT inside aggregates increases, and therefore, the number of alkyl chains (more hydrophobic than PPO chains) surrounding pyrene molecules also increases. The other reason might be a change in micellar structure, which would cause pyrene molecules to be closer to the alkyl chains, and consequently, the PPO chains would change to a less extended conformation. Both possibilities will be discussed in the following sections.

Fluorescence measurements were also carried out in diluted samples to study the formation of aggregates. In Fig. 4, the $I_{1} / I_{3}$ ratios start to decrease from a given amphiphile concentration, reflecting a decrease in the polarity surrounding the probe, from an aqueous environment $\left(\mathrm{I}_{1} / \mathrm{I}_{3} \sim 1.7\right)$ to a less polar one with $\mathrm{I}_{1} / \mathrm{I}_{3} \sim 1.3$, closer to that corresponding to PPO chains (see Fig. 3). Namely, the behaviour of $I_{1} / I_{3}$ is an indication of the formation of aggregates in which pyrene molecules are embedded; the onset of aggregation can be assigned to the first break point in the curves. The decrease in the $\mathrm{I}_{1} / \mathrm{I}_{3}$ ratios is not sharp as for single low molecular weight surfactants but takes place over a wide range of concentration (almost two orders of magnitude), which may indicate different stages in the association process and the presence of species with different molecular weights, as in the case of the block copolymers used in the present study.

The ratio of the fluorescence intensities of pyrene in the excimeric $\left(\mathrm{I}_{\mathrm{e}}\right)$ and monomeric $\left(\mathrm{I}_{3}\right)$ states provides information about the local concentration of pyrene in the aggregates [34]. As can be seen in Fig. 4, the $I_{e} / I_{3}$ curves show a maximum, a behaviour that has been reported previously [33]. At low concentrations, pyrene molecules are distributed in the bulk solution, and therefore, the $\mathrm{I}_{\mathrm{e}} / \mathrm{I}_{3}$ ratios are low. As the first aggregates are formed, there is a local increase in probe concentration as pyrene molecules migrate to such aggregates, which causes also an increase in $\mathrm{I}_{\mathrm{e}} / \mathrm{I}_{3}$ ratio. A further increase in amphiphile concentration results in a larger number of aggregates and therefore 

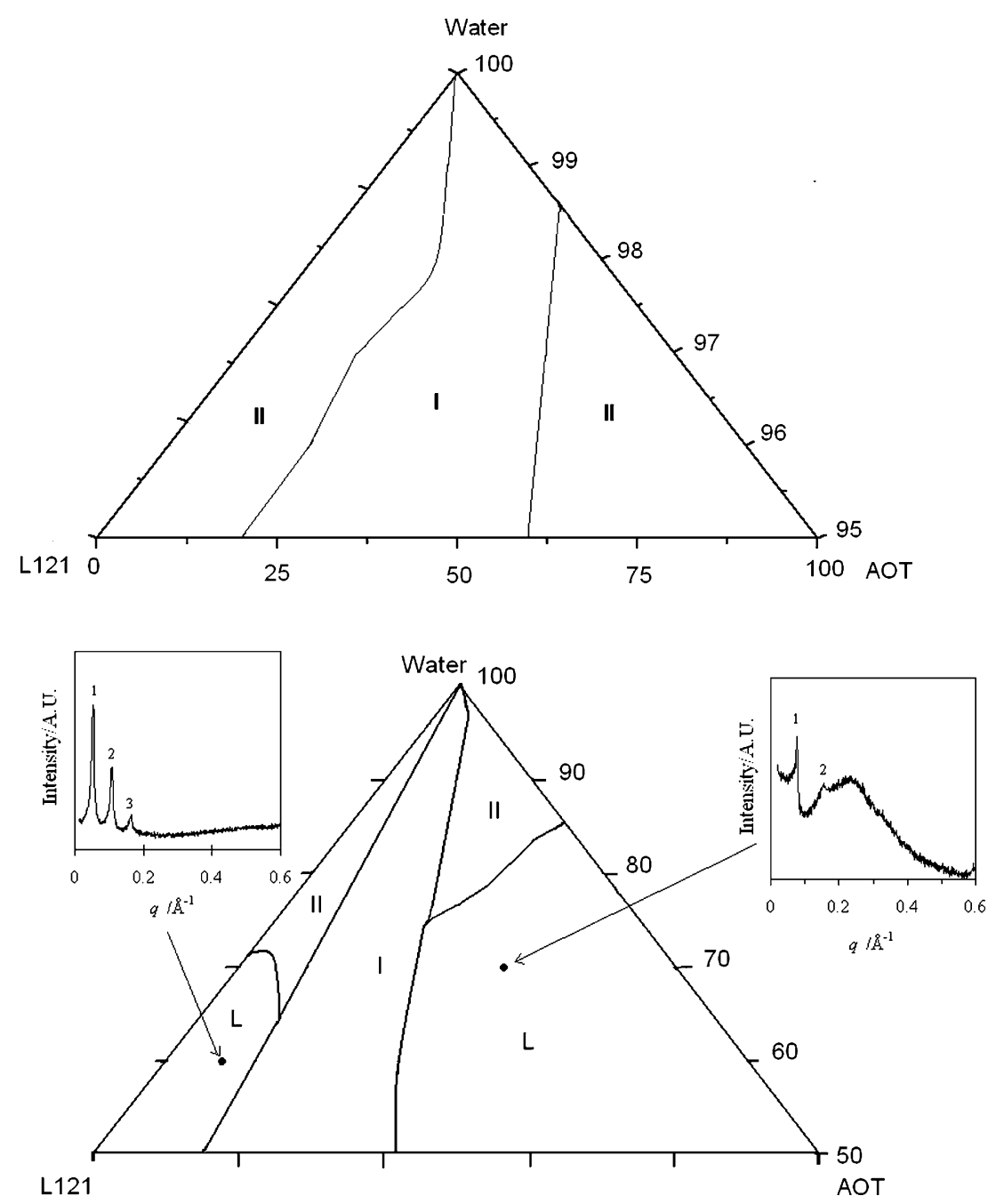

Fig. 2 Partial phase diagrams of AOT/L121/water system at $25^{\circ} \mathrm{C}$ in the dilute region (top) and concentrated (bottom) regions. $\mathrm{L}$ indicates a region in which lamellar liquid crystals are present. I and II indicates

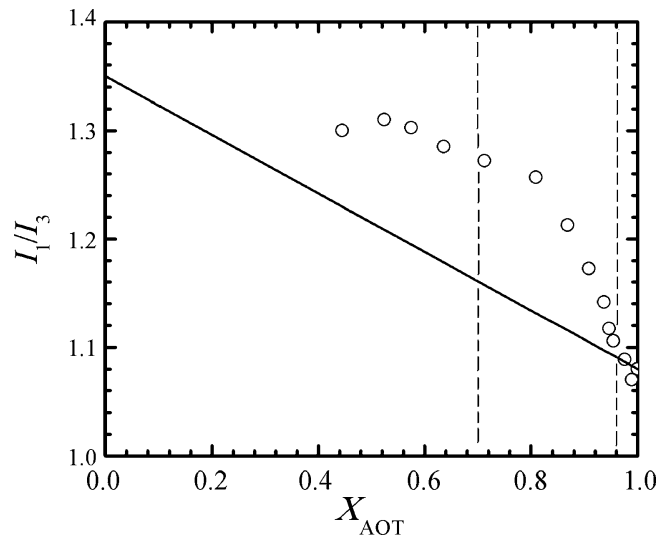

Fig. 3 Variation of the pyrene polarity ratio $I_{1} / I_{3}$ at $25^{\circ} \mathrm{C}$ as a function of AOT bulk mol fraction in the AOT $+\mathrm{L} 121$ mixture (solvent-free basis). The total amphiphilic concentration (AOT + L121) is kept constant at $5 \mathrm{wt} \%$. The continuous line represents a simple linear mixing relationship (Eq. 2). The dashed lines are the boundaries of the isotropic, single-phase region single and multiphase regions, respectively. The insets in the bottom are SAXS spectra of representative samples; position ratios are indicated on the peaks. $q$ is the scattering vector

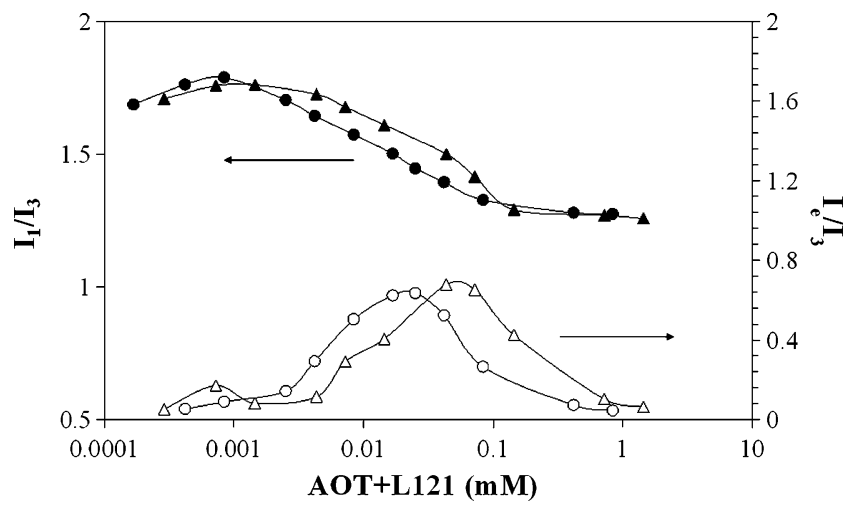

Fig. 4 Plot of $\mathrm{I}_{1} / \mathrm{I}_{3}$ (filled symbols) and $\mathrm{I}_{\mathrm{e}} / \mathrm{I}_{3}$ (open symbols) ratios as a function of total amphiphile concentration. Circles: $X_{A O T}=0.81$; Triangles: $X_{A O T}=0.94$ 
pyrene molecules will also be distributed in a larger volume; as a consequence, the $\mathrm{I}_{\mathrm{e}} / \mathrm{I}_{3}$ ratio decreases after the maximum. Contrary to previous results on single PEOPPO copolymer systems, in the present case, the $\mathrm{I}_{\mathrm{e}} / \mathrm{I}_{3}$ maximum does not coincide with the first break in the $I_{1} / I_{3}$ curves but more with the second, which suggests that in the present mixed system the singular points in each curve might indicate different aggregation states, as will be discussed in the next section.

\section{Critical aggregation concentration determination}

Surface tension measurements were used to determine the critical aggregation concentration $(c a c)$ of mixed AOT + L121 solutions. It should be emphasized here that for all experiments the same L121 product lot was used to reduce the effect of composition changes. Two breaks were observed in the surface tension isotherm (Fig. 5). The appearance of two breaks has been ascribed to the broad distribution of the molecular weight of polymer, a change of molecular conformation at the air/water interface, or the formation of two types of aggregates [35, 36]. There are reports on discrepancies between the results on cac of Pluronic solutions estimated by different techniques [37]. However, in the present case, the first break in Fig. 5 correspond to that found in $I_{1} / I_{3}$ curves of Fig. 4 , and the second break occurs at approximately the same concentration as that of the maximum in $\mathrm{I}_{\mathrm{e}} / \mathrm{I}_{3}$ curves in that figure. Therefore, fluorescence and surface tension results are in agreement in the present case. It is possible then that first AOT, L121 and pyrene molecules associate in some kind of structures that are more "open", so that the local pyrene concentration is not much different to that of the bulk solution but still the probe "feels" a different environment. This kind of association could be similar to the well-known pearl-necklace model for surfactant/polymer interactions [38]. The second kind of association will be closer to typical aggregates in which local pyrene concentration is

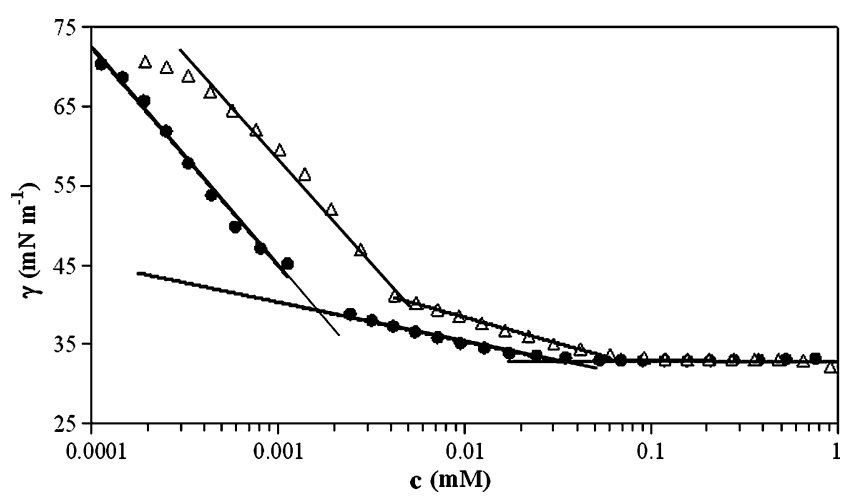

Fig. 5 Representative surface tension curves for two different $X_{A O T}$ values. The total amphiphilic concentration (AOT + L121) is kept constant at $5 \mathrm{wt} \%$. Circles: $X_{A O T}=0.81$; Triangles: $X_{A O T}=0.94$ different from that of the bulk. We followed the criteria of Alexandridis et al [35], and took the second break as the concentration corresponding to the formation of mixed micellar-like aggregates $\left(c a c^{*}\right)$.

The mixed critical aggregation concentration $\left(c a c^{*}\right)$ of an ideal surfactant mixture is given by [39]

$$
\frac{1}{c a c^{*}}=\frac{X_{A O T}}{\operatorname{cac}_{1}}+\frac{\left(1-X_{A O T}\right)}{\operatorname{cac}_{2}}
$$

where $X_{A O T}$ is the bulk mole fraction of surfactant 1 (AOT) in total mixed solute, and $c a c_{1}$ and $c a c_{2}$ are the critical aggregation concentrations of pure components 1 and 2, respectively. Fig. 6 shows that the $c a c$ values for AOT + L121 mixtures deviate negatively from the ideal $c a c^{*}$ values at medium to high AOT fractions. Such a negative deviation is related to favorable mixing, i.e., synergistic interaction between monomers of AOT and L121 in the mixture, as observed in other Pluronic-Ionic surfactant mixed systems [40].

The AOT mol fraction in aggregates $\left(x_{1}\right)$ can be estimated by solving the following equation derived from the regular solution theory pseudophase separation model [43]

$\frac{x_{1}^{2} \ln \left(X_{\text {AOT }} c a c^{*} / x_{1} \operatorname{cac}_{1}\right)}{\left(1-x_{1}\right)^{2} \ln \left[\left(1-X_{A O T}\right) c a c^{*} /\left(1-x_{1}\right) \operatorname{cac}_{2}\right.}=1$

where $\mathrm{cac}_{1}$ and $\mathrm{cac}_{2}$ are the cacs of AOT and L121, respectively. A plot of $x_{1}$ as a function of $X_{A O T}$ is presented in Fig. 7. In most range of AOT bulk fractions, L121 molecules are a majority inside the aggregates. Above $X_{A O T} \approx 0.8$, the mol fraction of AOT in aggregates (near the cac) sharply increases. This behavior is similar to that observed with fluorescence measurements at high surfactant concentrations, which also suggested an increase in the $x_{1}$ at about the same value for $X_{A O T}$. The values of interaction

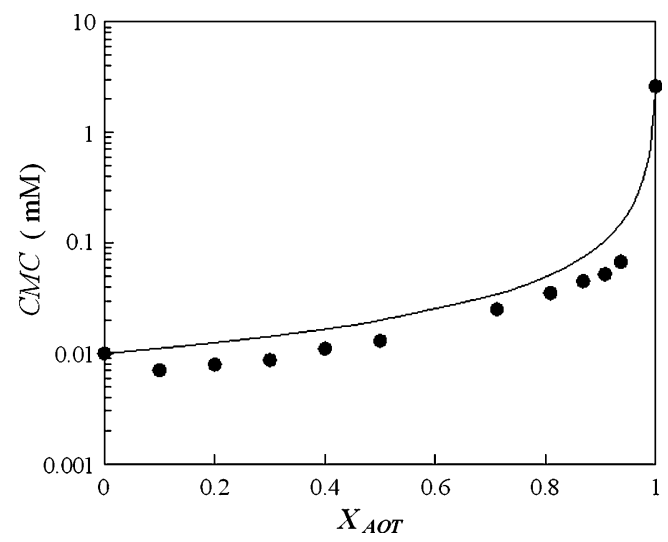

Fig. 6 Critical aggregation concentrations (cac) from surface tension measurements at $25^{\circ} \mathrm{C}$ as a function of AOT mol fraction in the AOT $+\mathrm{L} 121$ mixture (solvent-free basis). The total amphiphilic concentration (AOT $+\mathrm{L} 121)$ is kept constant at $5 \mathrm{wt} \%$. The line corresponds to an ideal mixture. The values for pure AOT and pure L121 were obtained from Ref. [41] and Ref. [42], respectively 
parameter $\beta$ derived from the application of the regular solution theory pseudo phase separation model [43] to the $c a c^{*}$ experimental data showed a large variation with composition, as can be observed in Fig. 7. $\beta$ is highly negative, reflecting the asymmetry of the system.

Small-angle neutron scattering (SANS) measurements

SANS curves from $5 \mathrm{wt} \%$ micellar solutions of L121 and AOT mixtures with varying AOT mole fractions in the $\mathrm{AOT}+\mathrm{L} 121$ mixture $\left(X_{\mathrm{AOT}}\right)$ at $25^{\circ} \mathrm{C}$ are shown in Fig. 8 . Some of the raw data were briefly introduced recently by some of the authors of the present paper [44]. All the distributions show well-defined peaks characteristic of suspensions of charged particles with strong repulsive intermicellar interactions. It is observed that an increase in the AOT/L121 ratios results in a large decrease in the scattering intensities and a shifting of the peak position to higher $Q$ values, reflecting a change in aggregate dimensions. It is to be noted here that the peak in SANS arises from a corresponding peak in the inter-particle structure factor $S(Q)$ the position of which at $Q_{m}(\approx 2 \pi / d)$ depends on the inter-particle distance $d$. Therefore, a shift in the peak position towards lower $Q$ values suggests an increase in micellar size and vice-versa. As a consequence, the present SANS results indicate that the mixed aggregates of AOT and L121 decrease in size as $X_{A O T}$ increases.

We used the radius of gyration as a fixed parameter, calculated [45] from the contour length $L$ and the Kuhn statistical segment length $k$ as $R_{\mathrm{g}}=(L k / 6)^{1 / 2}$. From the bond lengths and angles, the contour length per one ethylene oxide unit is $3.5 \AA$, while the Kuhn length for poly(ethylene oxide) is about $10 \AA$ [46]. From these values $R_{\mathrm{g}}=5 \AA$ is obtained for the hydrophilic chains of $5 \mathrm{EO}$ units, which agrees with other theoretical calculations [47] and values calculated by extrapolation of experimental data

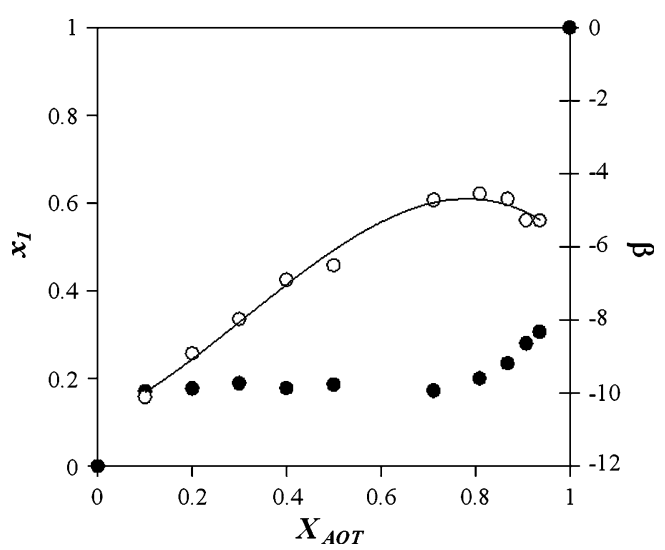

Fig. 7 Aggregate mole fraction of AOT ( $x_{1}$, filled circles) and interaction parameters $(\beta$, open circles) versus bulk mole fraction $\left(X_{\mathrm{AOT}}\right)$ in L121 + AOT aqueous systems derived from data in Fig. 6. The line is the best fit to a cubic polynomial of longer chains [48, 49]. The fitting model would be similar to a "crew-cut" morphology [50], with large hydrophobic cores and relative thin hydrophilic coronas. We cannot completely rule out that part of PPO chains are also hydrated, which would increase the value for $R_{\mathrm{g}}$. In any case, the parameters in Table 1 changed only slightly upon varying $R_{\mathrm{g}}$.

The values obtained from the fit are shown in Table 1, and they correspond to relatively small aggregates. Absolute values of the parameters for prolate ellipsoids should be taken with precaution, as the fitting of the theoretical model is far from perfect; however, data have been included to compare with that coming from the spherical model. The estimated values for the axial ratio $(a / b)$ were also relatively small (1.7-1.8) and change very little with $X_{A O T}$. The studied samples showed low viscosity, which rules out the presence of highly elongated or entangled aggregates [51]. Cryo-TEM pictures (not shown) did not give evidence of very long aggregates either. In fact, and as can be seen in

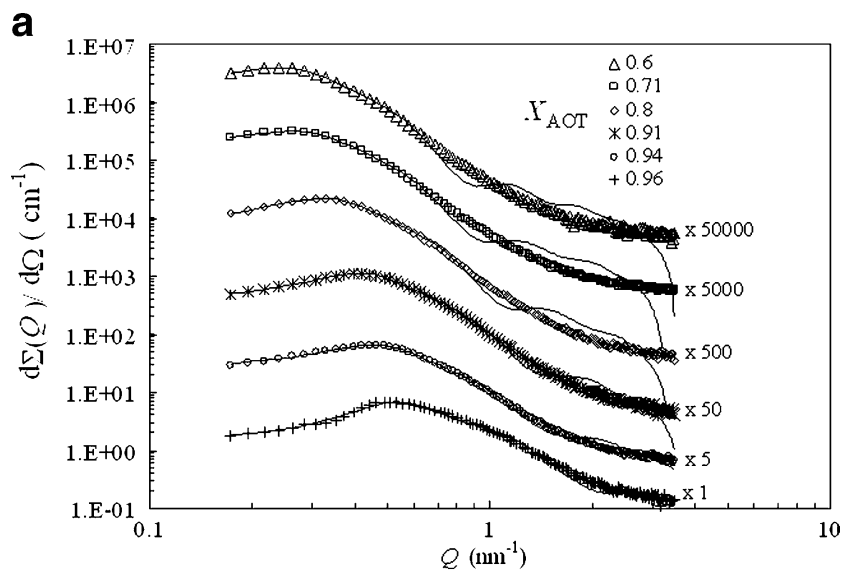

b

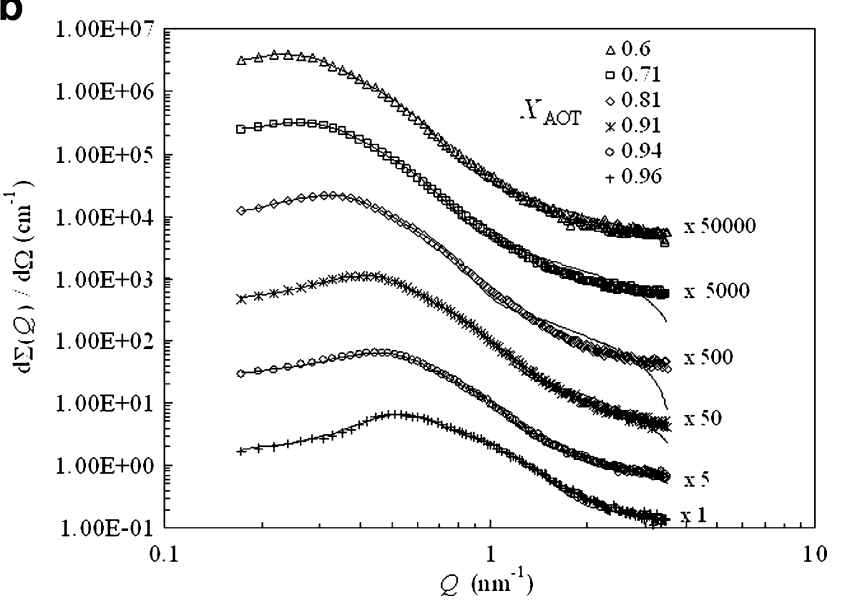

Fig. 8 SANS data for AOT+L121 solutions at various AOT mole fractions $\left(X_{\mathrm{AOT}}\right)$ and the corresponding fitted curves (solid lines) for prolate ellipsoids (a), and polydisperse spheres (b). The total amphiphile concentration (AOT $+\mathrm{L} 121$ ) is kept constant at $5 \mathrm{wt} \%$. Curves have been multiplied by the indicated factors for clarity of presentation 
Fig. 8, experimental data were better fitted by the spherical model with a polydispersity close to 0.2 , a value similar to that reported for other pluronic-ionic surfactant systems $[13,52]$.The sizes estimated from SANS are larger than those derived from Dynamic Light Scattering in our previous report [18], but there is an agreement in the decreasing tendency of the size with increasing $X_{A O T}$.

According to an earlier report [53], AOT aggregates at $1 \mathrm{wt} \%$ in water have the shape of prolate ellipsoids with $a=$ $20.6 \AA$ and $b=12.6 \AA$ (axial ratio=1.64); namely, addition of L121 induces micellar swelling. Since the hydrodynamic radius of $\mathrm{L} 121$ unimers in water (at $10^{\circ} \mathrm{C}$ ) is $19 \AA$ [54], the copolymer seems to be in a more extended conformation when incorporated into the mixed aggregates, namely, the AOT hydrophobic chains would be acting as a relatively good solvent for PPO. In fact, we found that PPO with the same molecular weight of the PPO block of L121, and octane (similar to the alkyl chain of AOT) are mutually soluble in all proportions, which could be expected from the proximity of their solubility parameters.

Table 1 also shows that the fractional charge $(\alpha)$ increases with AOT content, as expected. The $\alpha$ values are higher in the case of the spherical model, and are comparable to those reported for pure AOT micelles in water [53]. No major changes in the size and geometry of aggregates were observed when temperature was increased from 30 to $50^{\circ} \mathrm{C}$ (see supplementary material (ESM 1)).

It is apparent that there is an optimum range of AOT/ L121 mixing ratios for the formation of quasispherical

Table 1 Structural parameters of mixed micellar aggregates obtained from the SANS distribution

\begin{tabular}{|c|c|c|c|c|}
\hline$X_{\text {АОт }}$ & $b^{\mathrm{a}}(\AA)$ & $r^{\mathrm{b}}(\AA)$ & $\alpha^{\mathrm{c}}(e-/$ molecule $)$ & Aggregation number \\
\hline \multicolumn{5}{|c|}{ Model: Prolate ellipsoid ${ }^{\mathrm{d}}$} \\
\hline 0.60 & 48 & & 0.12 & 248 \\
\hline 0.71 & 44 & & 0.12 & 250 \\
\hline 0.81 & 37 & & 0.18 & 187 \\
\hline 0.91 & 29 & & 0.24 & 131 \\
\hline 0.94 & 26 & & 0.26 & 108 \\
\hline 0.96 & 20 & & 0.45 & 53 \\
\hline \multicolumn{5}{|c|}{ Model: polydisperse spheres ${ }^{\mathrm{d}}$} \\
\hline 0.60 & & 44 & 0.29 & 111 \\
\hline 0.71 & & 42 & 0.27 & 114 \\
\hline 0.81 & & 37 & 0.33 & 104 \\
\hline 0.91 & & 29 & 0.42 & 75 \\
\hline 0.94 & & 26 & 0.48 & 60 \\
\hline 0.96 & & 19 & 0.92 & 29 \\
\hline
\end{tabular}

${ }^{\text {a }}$ Semi-minor axis

${ }^{\mathrm{b}}$ Sphere radius

c Fractional charge

${ }^{\mathrm{d}}$ Total $($ AOT $+\mathrm{L} 121)$ concentration $=5 \mathrm{wt} \%$ aggregates. For $X_{A O T}<0.7$ (low concentration boundary of the single phase in Fig. 1), there is not enough AOT to solubilize the hydrophobic PPO chains, therefore, phase separation is favoured. For $X_{A O T}>0.94$ (high concentration boundary of the single phase in Fig. 1), the entropy penalty derived from the compression of the PPO chains within the small aggregates would be too high and again, the system would tend to phase separate. Trying to get more insight one the role of L121 on the phase transitions, we observed the phase behaviour at $25^{\circ} \mathrm{C}$ of aqueous mixtures of AOT with PPO with the same molecular weight as that of the L121 hydrophobic block, keeping the total concentration as that used in the AOT+L121 system (i.e., $5 \mathrm{wt} \%$ ). We found a single phase isotropic region, but much narrower than that for AOT+L121 systems (between $X_{A O T}=0.5$ and $X_{A O T}=0.7$ ); namely, L121 does not simply act as a swelling hydrophobe.

According to an earlier article by Fan et al. [55], in mixtures of AOT with pentaethylenglycol dodecyl ether $\left(\mathrm{C}_{12} \mathrm{E}_{5}\right)$, only vesicles are formed and no vesicle-micelle transition was detected. Since L121 and $\mathrm{C}_{12} \mathrm{E}_{5}$ have the same PEO chain length (L121 in both ends of the molecule), it seems that L121 long hydrophobic chain is determinant for the vesiclemicelle transition. The critical packing parameter [56] $P=$ $v /(S r)$, where $v$ and $r$ are the molar volume and length of the amphiphile hydrophobic moiety, respectively, and $S$ is the effective surface area per surfactant molecule, is also appropriate for a qualitative analysis of the results. For single L121 or AOT molecules in water, $S$ is small and $v$ relatively high, therefore structures with low curvature and high $P$, such as vesicles, are favoured. In order to have quasispherical aggregates with $P \approx 1 / 3$, either high $S$ or high $r$ values are needed. In the present case, there is swelling of the micellar cores by the solubilization of PPO chains (increase of effective $r$ ) while relatively high $S$ values are induced by the electrostatic repulsion between AOT head groups. Hence, this synergism is the driving factor for the formation of quasispherical aggregates.

We have also performed SANS measurements for $0.5 \mathrm{wt} \%$ solutions of L121 and AOT mixtures as shown in Fig. 9, so as to keep the micellar volume fraction low and thereby minimize intermicellar interactions (the contribution of structure factor was neglected in the calculations).

With the increase in $X_{A O T}$ value, there is a decrease in the low- $Q$ scattering while the intensity at higher $Q$ regions $\left(Q>2 \mathrm{~nm}^{-1}\right)$ remains practically unchanged. The decrease in low- $Q$ scattering is again consistent with the decrease in the micellar size with the increase in fraction of AOT, which is clearly observed when comparing the micellar volumes (see Table 2). However, the scattering patterns at low concentrations corresponded to disk-like aggregates, indicating a shape transition from short prolate ellipsoids (or spheres) to disks upon dilution. Such a transition also results in an increase in the aggregation numbers (see Table 2). At 


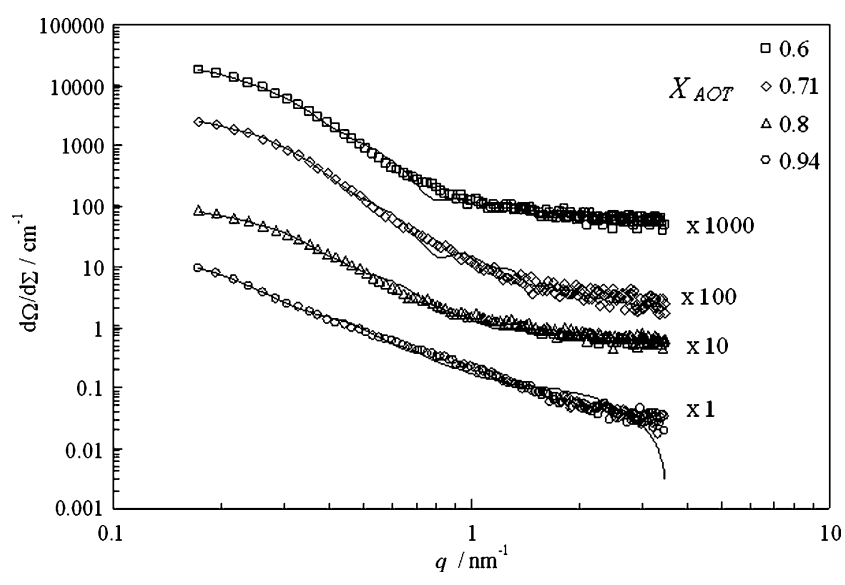

Fig. 9 SANS data for AOT + L121 solutions at various AOT mole fractions $\left(X_{\text {АОт }}\right)$ and the corresponding fitted curves (solid lines) for disks. The total amphiphile concentration (AOT + L121) is kept constant at $0.5 \mathrm{wt} \%$. Curves have been multiplied by the indicated factors for clarity of presentation

$0.5 \mathrm{wt} \%$, single AOT in water has been reported to have ellipsoidal shape [53] whereas single L121 forms vesicles or lamellar structures [54]; namely, again there is a morphological change when the single surfactant systems are mixed.

\section{DSC measurements}

The DSC trace of L121 in water, Fig. 10, shows an endothermic peak having a small shoulder, with an onset temperature $14^{\circ} \mathrm{C}$ and an associated enthalpy of $474 \mathrm{~kJ} /$ gmol. The presence of this composite peak is attributed to the formation of vesicles or a dispersion of a lamellar liquid crystal in a two-step process [57]. It is known that the enthalpy change of pure L121 solutions is related with the aggregation process, and results from the dehydration of PPO chains [57]. Below a certain temperature (i.e. $14^{\circ} \mathrm{C}$ ), L121 is in a monomeric state. Note that such state can not be observed in the phase diagram of Fig. 2 since it was constructed at $25^{\circ} \mathrm{C}$. When AOT is added the onset of the peak is shifted to lower temperatures and the enthalpy associated with the peak decreases; peak broadening is also observed. Therefore, adding AOT induces the formation of aggregates at lower temperatures. Such a decrease in aggregation temperature and enthalpy of aggregation has also been found when nonionic surfactants are added to block copolymers $[58,59]$. On the other hand, the shoulder observed in the DSC curve of L121 aqueous solution also disappears upon addition of AOT, which suggest that the nature of the transition is different. Actually, the formation of micelles in other Pluronic aqueous solutions is reflected by a single peak, as in the present case when AOT is added. In L121 + sodium dodecyl sulfate (SDS) mixed systems, the micellization enthalpy also decreases upon adding the ionic surfactant, but the onset temperature actually increases [57]. AOT is much less hydrophilic than SDS, and therefore the effect should be different. It seems that AOT favours the formation of mixed aggregates with L121 at low temperatures. The decrease in the aggregation enthalpy upon addition of AOT is clearly observed in Fig. 10 (bottom) and could be attributed to the formation of smaller aggregates and to a decrease of the dehydration enthalpy as the fraction of PPO chains in the aggregates decreases, which supports SANS results.

Results on ITC measurements are presented in Fig. 11. In Fig. 11a, the curve for pure AOT (enthalpogram) shows a sigmoidal shape. Initially, the surfactant undergoes disaggregation followed by monomer dilution. Heat changes result from the disaggregation of surfactant aggregates and dilution of monomers. At the state of aggregation and above, the solution undergoes aggregate dilution (and not further disaggregation) with an appreciable change in the enthalpy that remains nearly constant with increasing surfactant interaction [60]. The difference between the final and initial part of enthalpogram corresponds to the enthalpy of aggregation, $\Delta \mathrm{H}_{\mathrm{agg}}$, as it is shown in Fig. 11a. The obtained value of $3.68 \mathrm{~kJ} / \mathrm{mol}$ is slightly higher from the value of $3.12 \mathrm{~kJ} / \mathrm{mol}$ obtained by calorimetry by Chattterjee et al. [41].

The aggregation concentration is determined according to the Phillip's criterion [61] from the first derivative of the reaction heat against surfactant concentration (Fig. 11a). Estimated value of $2.55 \mathrm{mM}$ is in good agreement with the values $(2.72 \mathrm{mM}, 2.68 \mathrm{mM})$ reported in the literature [41].

The calorimetric behavior is very much different for mixed AOT + L121 systems (Fig. 11b). Here curves do not present clear sigmoidal features and were analyzed by a data treatment as it has been described by Bouchemal et al recently [62].

The start of transition (ST) and end of transition (ET) has been determined as it is shown in Fig. $11 \mathrm{~b}$ for $X_{A O T}=0.3$. Linear fits of the data sets in the lower and upper concentration domains were performed and the intercepts of the two straight lines were determined. The difference between this two intercepts yields the enthalpy of the transition (aggregation). This procedure yields values of about $60 \pm 5 \mathrm{~kJ} / \mathrm{mol}$ and $180 \pm 10 \mathrm{~kJ} / \mathrm{mol}$ for the surfactant

Table 2 Structural parameters of mixed micellar aggregates obtained from the SANS distribution

\begin{tabular}{lcll}
\hline $\mathrm{X}_{\mathrm{AOT}}$ & Radius $(\AA)$ & height $(\AA)$ & Aggregation number \\
\hline 0.60 & 96 & 73 & 633 \\
0.71 & 92 & 76 & 768 \\
0.80 & 91 & 50 & 627 \\
0.94 & 127 & 44 & 1863 \\
\hline
\end{tabular}

Model: Disk. Total $(\mathrm{AOT}+\mathrm{L} 121)$ concentration $=0.5 \mathrm{wt} \%$ 

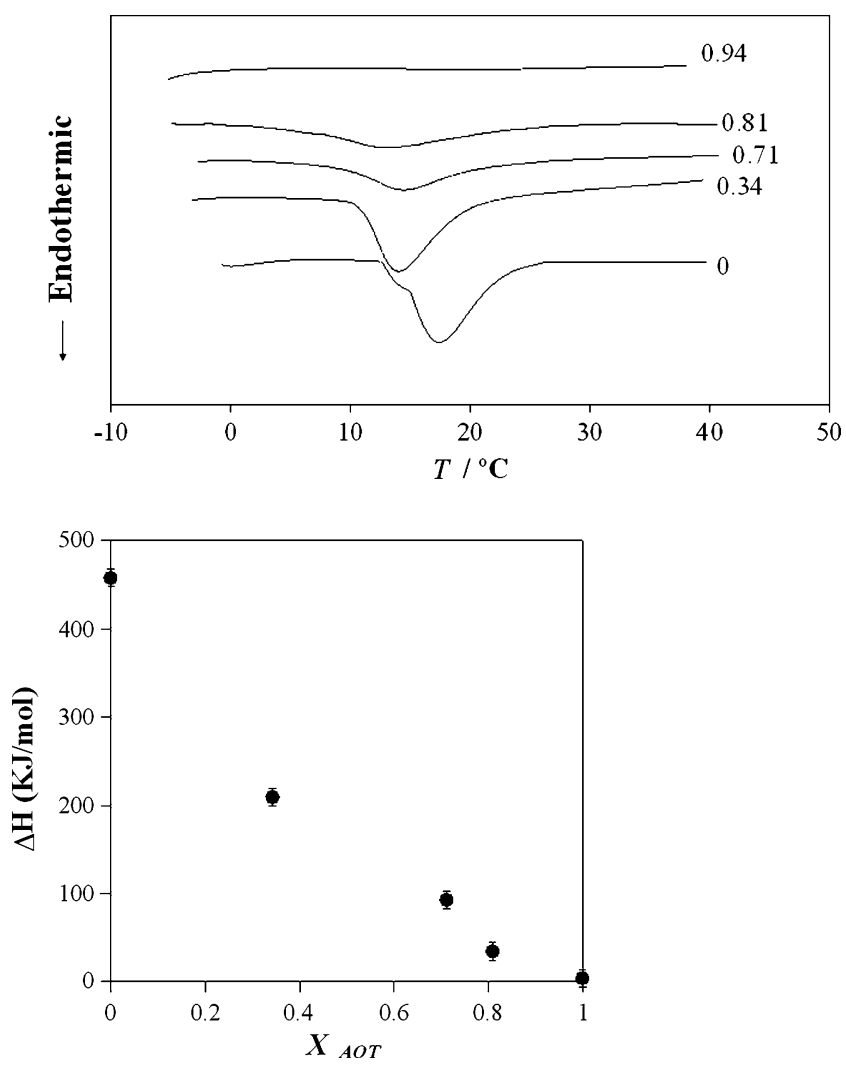

Fig. 10 DSC traces of AOT-L121 aqueous mixtures. $X_{\mathrm{AOT}}$ values are shown besides each curve (top). Transition enthalpies derived from DSC curves (bottom). The total surfactant concentration is $5 \mathrm{wt} \%$. The data for $\mathrm{XAOT}=1$ is taken from ITC measurements (see discussion below)

mixtures with $X_{A O T}=0.6$ and 0.3 , respectively. As it can be seen from Fig. 10 they are slightly lower from those obtained by DSC technique, but the difference can be ascribed to the error made by the linear fits and the extrapolation of the ITC data, although kinetic effects cannot be completely ruled out. Note that aggregation takes place at very low concentrations, so that the locus of the transition from monomer to aggregates cannot be observed in the phase diagram of Fig. 2a.

Nevertheless, the enthalpy change increases as $X_{A O T}$ decreases, i.e. as L121 content increases. Actually, the shape of the curves suggest the presence of at least two processes (indicated by the breaks in the straight dotted lines in Fig.11b), in qualitative agreement with the surface tension results shown in Fig. 4.

\section{Dynamic surface tension measurements}

Dynamic surface tension (DST) measurements were carried out as a function of $X_{\mathrm{AOT}}$ at different total surfactant concentrations ( $5 \mathrm{wt} \%, 0.5 \mathrm{wt} \%$, and $0.005 \mathrm{wt} \%$ ) at $25^{\circ} \mathrm{C}$. At high total surfactant concentrations (e.g. $5 \mathrm{wt} \%$ and $0.5 \mathrm{wt} \%)$, the DST data showed almost no change with time and the values were close to those at equilibrium (see supporting material). At lower total surfactant concentration (0.005 wt $\%)$, the DST curves are different: an induction region (at short times), followed by a decreasing region are clearly visible in the DST curves, see Fig. 12 (top). Usually, the reduction of dynamic surface tension involves several mechanisms and a typical surface tension vs time curve can be divided into four regions [63]: an induction region (short times), a rapid fall region, a mesoequilibrium region, and an equilibrium region (at a long time). However, we have observed only the first two regions for the dilute samples. A relatively long induction time $(\sim 1000 \mathrm{~ms})$ can be attributed to the low $c m c$ of the systems $[64,65]$. When a new interface is created, the surface tension is high but as the time passes the adsorption of surfactant molecules at the gas-liquid interface cause a decrease in the surface tension. Minute observation of the DST curves reveals that with increasing mixing fraction of AOT $\left(X_{\mathrm{AOT}}\right)$ the induction region tends to shorten indicating a faster diffusion to the surface.

We define a normalized dynamic surface tension $\gamma_{n}=\left(\gamma_{\mathrm{t}}-\gamma_{\infty}\right) /\left(\gamma_{\mathrm{o}}-\gamma_{\infty}\right)$, where $\gamma_{\mathrm{t}}, \gamma_{\mathrm{o}}$ and $\gamma_{\infty}$ are the

a
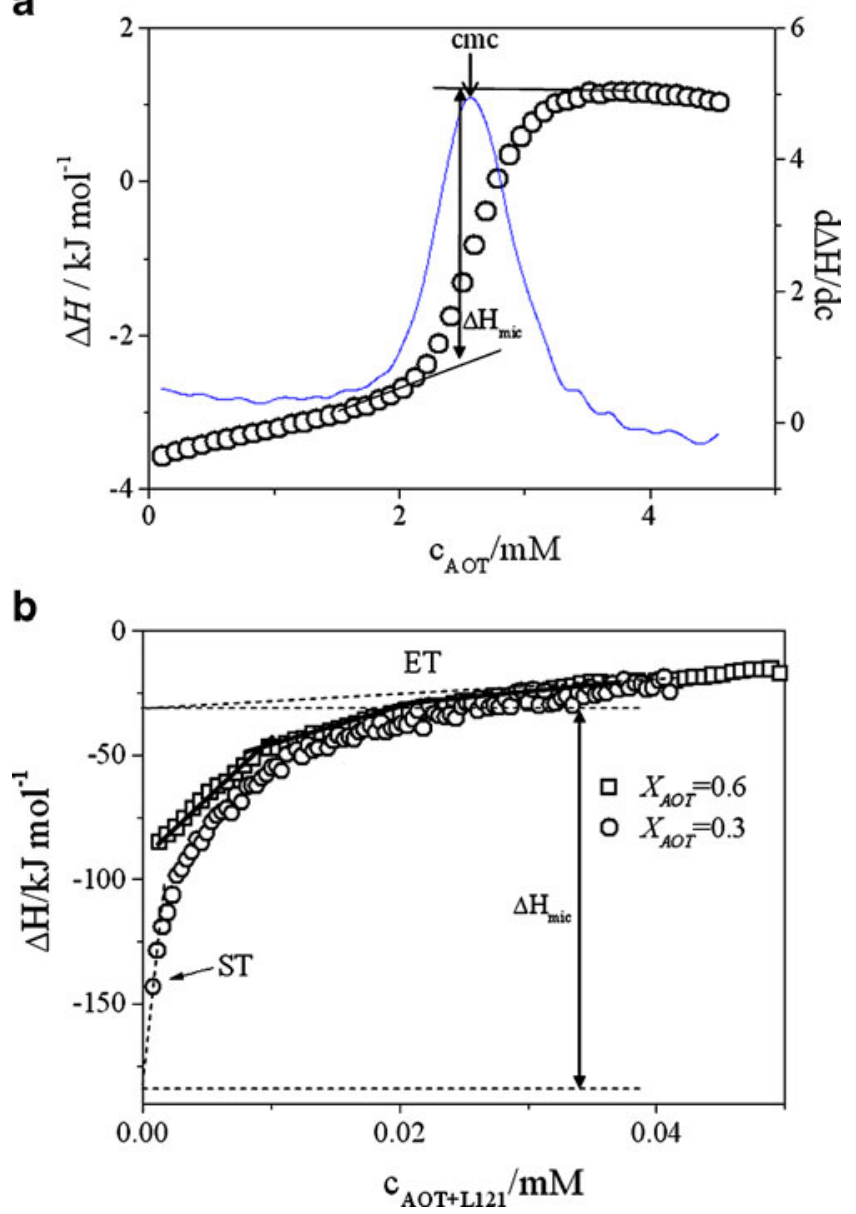

Fig. 11 Reaction heat as a function of total amphiphile concentration in $\mathrm{AOT}$ (a) and AOT $+\mathrm{L} 121$ (b) aqueous systems at $25^{\circ} \mathrm{C}$ 
surface tensions at time $t$, at $t \rightarrow 0$ and at equilibrium, respectively. The following equation is used for fitting the experimental data [64]

$\gamma_{n}=\sum_{i=1}^{n} a_{i} \exp \left(-t / \tau_{i}\right)$

where $a_{\mathrm{i}}$ is a constant and $\tau_{i}$ is a relaxation time. In the present case, the experimental data could be fitted to a single exponential with a single relaxation time, which is plotted in Fig. 12 (bottom). The normalized DST and their exponential fit are supplied as supplementary material (ESM 1). The decrease of relaxation time with increasing $X_{\mathrm{AOT}}$ also suggests the faster diffusion of surfactant molecules to the surface.

Oil solubilization

To further explore the properties of mixed L121+AOT aggregates, solubility studies of a model oil were carried out. As it is shown in Fig. 13, the aromatic hydrocarbon $p$ xylene can be solubilized in the isotropic region of Fig. 1, which is the typical of micellar aggregates with a swollen core. The amount of solubilized oil increases with the AOT
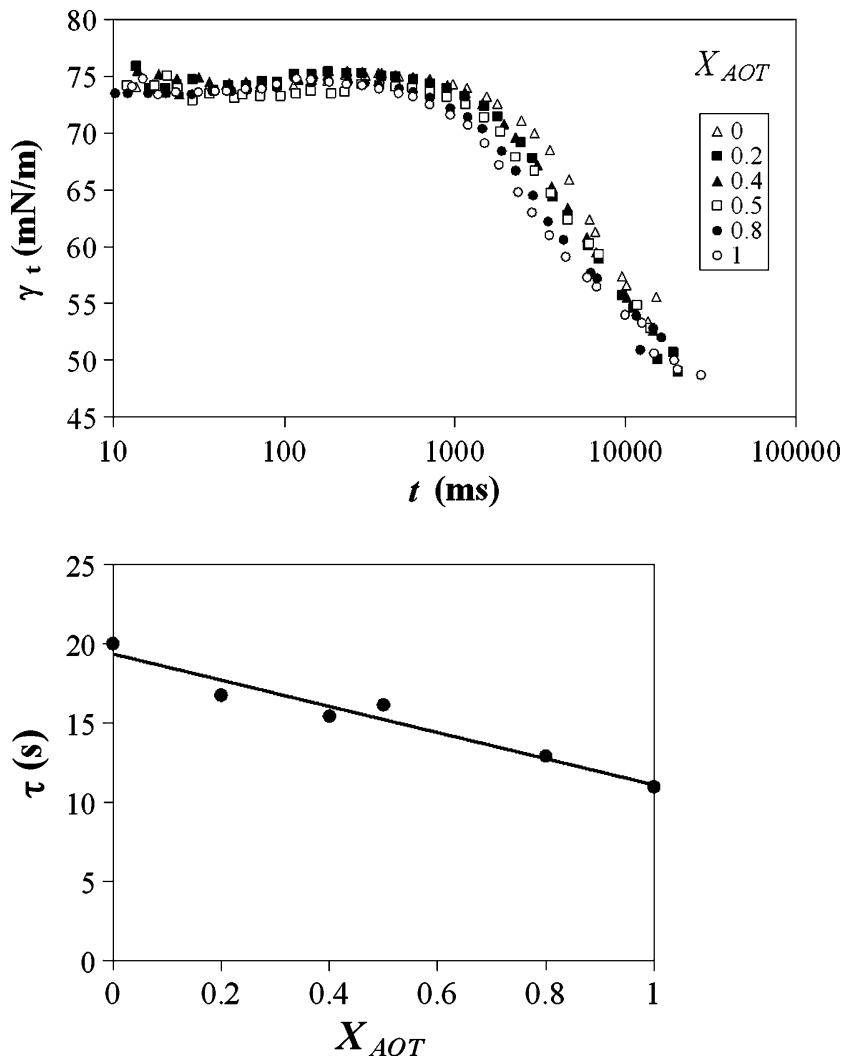

Fig. 12 Dynamic surface tensions at $25^{\circ} \mathrm{C}$ for different values of $X_{A O T}$ in $\mathrm{AOT}+\mathrm{L} 121$ aqueous mixtures (top), and relaxation time as a function of $X_{A O T}$ from the fitting of data in (a) using Eq. 5 (bottom). The surfactant concentration is fixed at $0.005 \mathrm{wt} \%$

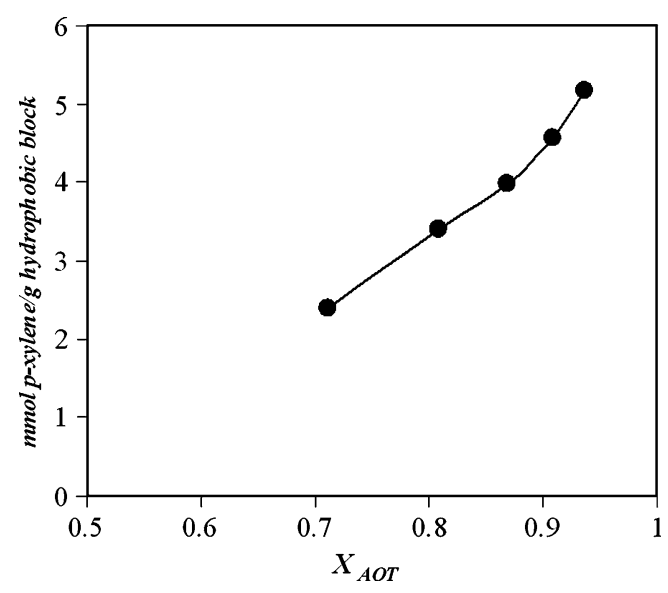

Fig. 13 Solubilization of $p$-xylene in AOT $+\mathrm{L} 121$ aqueous mixtures at $25^{\circ} \mathrm{C}$. The total amphiphilic concentration (AOT + L121) is kept constant at $5 \mathrm{wt} \%$. The line is only a visual guide

fraction. Mixing with L121 increases the micellar solubilization of $p$-xylene with respect to the binary AOT + water systems [65]. At $X_{\mathrm{AOT}}>0.85$, the solubilization capacity of the mixtures is higher than that of SDS or that of other pluronics with the same hydrophobic chain length as L121 [66]. According to the theory of Nagarajan [66], it could be explained in terms of a favourable change in the solubility parameters of the mixture with respect of that of the solubilizate ( $p$-xylene), namely, there is a synergistic effect.

\section{Conclusions}

A bilayer-micelle-bilayer transition takes place in a mixture of two hydrophobic amphiphiles, Pluronic L121 and AOT, which results in the formation of a transparent isotropic solution when mixing two turbid dispersions. Experimental evidence from SANS points to the existence of relatively small, quasispherical aggregates in the isotropic solutions, which decrease in size when the AOT fraction is increased; however, upon dilution, the aggregates change to disk-like. The inner core of aggregates is occupied by the PPO hydrophobic chains of the copolymer that seem to be in an extended confirmation. Such a conformation, together with the repulsion between charged AOT groups, leads a decrease in the effective packing parameter, thus favouring the formation of quasispherical aggregates. This synergistic effect is also reflected in a faster adsorption at the airsolution interface and improved solubilisation capacity.

Acknowledgments Authors are grateful to Dr. Sylvain Prévost (Helmholtz Zentrum Berlin (HZB) für Materialien und Energie, Berlin, Germany) for his help in the analysis of SANS data and Dr. Alfredo González-Pérez (Lund University, Sweden) for CryoTEM observations. Authors also wish to thank Prof. Arturo LopezQuintela (Universidad Santiago de Compostela, Spain) for useful comments and Ana Maria Moreno (CSIC) for her help in the experiments. 
C.R. is also grateful to the Ministerio de Ciencia e Innovación, Spain (Project CTQ2008-01979/BQU) for financial support.

\section{References}

1. Chu B (1995) Structure and dynamics of block copolymer colloids. Langmuir 11:414-421

2. Alexandridis P, Holzwarth JF (2000) Block copolymers. Curr Opin Colloid Interface Sci 5:312-313

3. Hamley IW (1998) The physics of block copolymers. Oxford University Press, Oxford

4. Hadijichristidis N, Pispas S, Floudas GA (2003) Block copolymers. Wiley, New York

5. Tan Y, Srinivasan S, Choi KS (2005) Electrochemical deposition of mesoporous nickel hydroxide films from dilute surfactant solutions. J Am Chem Soc 127:3596-3604

6. Suzuki K, Ikari K, Imai H (2004) Synthesis of silica nanoparticles having a well-ordered mesostructure using a double surfactant system. J Am Chem Soc 126:462-463

7. Discher DE, Eisenberg A (2002) Polymer vesicles. Science 297:967-973

8. Hu Z, Jonas AM, Varshney SK, Gohy JF (2005) Dilution-induced spheres-to-vesicles morphological transition in micelles from block copolymer/surfactant complexes. J Am Chem Soc 127:6526-6527

9. Chen D, Li Z, Yu C, Shi Y, Zhang Z, Tu B, Zhao D (2005) Nonionic block copolymer and anionic mixed surfactants directed synthesis of highly ordered mesoporous silica with bicontinuous cubic structure. Chem Mater 17:3228-3234

10. Lu Q, Bazuin CG (2005) Solvent-assisted formation of nanostrand networks from supramolecular diblock copolymer/surfactant complexes at the air/water interface. Nano Lett 5:1309-1314

11. Bronich TK, Ouyang M, Kabanov VA, Eisenberg A, Szoka FC Jr, Kabanov AV (2002) Synthesis of vesicles on polymer template. J Am Chem Soc 124:11872-11873

12. Hecht E, Hoffmann H (1994) Interaction of ABA block copolymers with ionic surfactants in aqueous solution. Langmuir 10:86-91

13. Thurn T, Couderc S, Sidhu J, Bloor DM, Penfold J, Holzwarth JF, Wyn-Jones E (2002) Study of mixed micelles and interaction parameters for ABA triblock copolymers of the type EOm-POn -EOm and ionic surfactants: equilibrium and structure. Langmuir 18:9267-9275

14. Tulpar A, Tilton RD, Walz JY (2007) Synergistic effects of polymers and surfactants on depletion forces. Langmuir 23:4351-4357

15. Svitova TF, Radke CJ (2005) AOT and Pluronic F68 coadsorption at fluid/fluid interfaces: a continuous-flow tensiometry study. Ind Eng Chem Res 44:1129-1138

16. Postmus BR, Leermakers FAM, Koopal LK, Cohen-Stuart MA (2007) Competitive adsorption of nonionic surfactant and nonionic polymer on silica. Langmuir 23:5532-5540

17. Chen D, Li Z, Wan Y, Tu X, Shi Y, Chen Z, Shen W, Yu C, Tu B, Zhao D (2006) Anionic surfactant induced mesophase transformation to synthesize highly ordered large-pore mesoporous silica structures. J Mater Chem 16:1511-1519

18. Rodríguez C, Shrestha LK, López-Quintela MA (2007) Unusual formation of small aggregates by mixing giant multilamellar vesicles. J Colloid Interface Sci 312:108-113

19. Deo N, Somasundaran P (2003) Effects of sodium dodecyl sulfate on mixed liposome solubilization. Langmuir 19:7271-7275

20. Ollivon M, Lesieur S, Grabielle-Madelmont C, Paternostre M (2000) Vesicle reconstitution from lipid-detergent mixed micelles. BBA-Biomembranes 1508:34-50

21. Cohen-Stuart MA, Boekema EJ (2007) Two distinct mechanisms of vesicle-to-micelle and micelle-to-vesicle transition are mediated by the packing parameter of phospholipid-detergent systems. BBA-Biomembranes 1768:2681-2689

22. Hristova K, Kenworthy A, McIntosh TJ (1995) Effect of bilayer composition on the phase behavior of liposomal suspensions containing poly(ethylene glycol)-lipids. Macromolecules 28:76937699

23. Schalchli-Plaszczynski A, Auvray L (2002) Vesicle-to-micelle transition induced by grafted diblock copolymers. Eur Phys J E 7:339-344

24. Yan Y, Hoffmann H, Drechsler M, Talmon Y, Makarsky E (2006) Influence of hydrocarbon surfactant on the aggregation behavior of silicone surfactant: observation of intermediate structures in the vesicle-micelle transition. J Phys Chem B 110:5621-5626

25. Aswal VK, Goyal PS (2000) Small-angle neutron scattering diffractometer at Dhruva reactor. Curr Sci 79:947-953

26. Goyal PS, Aswal VK (2001) Micellar structure and inter-micelle interactions in micellar solutions: results of small angle neutron scattering studies. Curr Sci 80:972-979

27. Chen SH, Lin TL (1987) Colloidal solutions. In: Price DL, Skold $\mathrm{K}$ (eds) Methods of experimental physics-neutron scattering, Chapter 16. Academic Press, New York

28. Hayter JB, Penfold J (1983) Determination of micelle structure and charge by neutron small-angle scattering. Colloid Polym Sci 261:1022-1030

29. Aramaki K, Akahane A, Kunieda H (2000) Oil-induced phase transition from lamellar to reverse hexagonal liquid crystals in the Aerosol OT system Prog. Colloid Polym Sci 115:40-43

30. Svensson B, Olsson U, Alexandridis P (2000) Self-Assembly of block copolymers in selective solvents: influence of relative block size on phase behavior. Langmuir 16:6839-6846

31. Skouril M, Marignanl J, May R (1991) X-ray and neutron-scattering study of the lamellar and $\mathrm{L}_{3}$ phases of the system aerosol-OT-water: effect of NaCI and decane. Colloid Polym Sci 269:929-937

32. Alexandridis P, Nivaggioli T, Hatton TA (1995) Temperature effects on structural properties of Pluronic P104 and F108 PEOPPO-PEO block copolymer solutions. Langmuir 11:1468-1476

33. Bohorquez M, Koch C, Trygstad T, Pandit N (1999) A study of the temperature-dependent micellization of Pluronic F127. J Colloid Interface Sci 216:34-40

34. Turro N, Kuo P (1986) Pyrene excimer formation in micelles of nonionic detergents and of water-soluble polymers. Langmuir 2:438442

35. Alexandridis P, Athanassiou V, Fukuda S, Hatton TA (1994) Surface activity of poly(ethylene oxide)-block-poly(propylene oxide)-blockpoly(ethylene oxide) copolymers. Langmuir 10:2604-2612

36. De Lisi R, Milioto S (2000) Poly(ethylene oxide) 13 -poly(propylene oxide) $)_{30}$-poly(ethylene oxide) $)_{13}$ electrolyte interactions in aqueous solutions at some temperatures. Langmuir 16:5579-5583

37. Loh W (2006) Block copolymer micelles. In: Somasundaran P, Hubbard A (eds) Encyclopedia of surface and colloid science, 2nd edn. Taylor \& Francis, London

38. Lee LT, Cabane B (1997) Effects of surfactants on thermally collapsed poly(N-isopropylacrylamide). Macromolecules 30:6559-6566

39. Clint JH (1975) Micellization of mixed nonionic surface active agents. J Chem Soc Faraday Trans 71:1327-1334

40. Bakshi MS, Sachar S, Singh K, Shaheen A (2005) Mixed micelle behavior of Pluronic L64 and Triton X-100 with conventional and dimeric cationic surfactants. J Colloid Interface Sci 286:369-377

41. Borbely S (2000) Aggregate structure in aqueous solutions of Brij-35 nonionic surfactant studied by small-angle neutron scattering. Langmuir 16:5540-5545

42. Holland PM, Rubingh DN (1983) Nonideal multicomponent mixed micelle model. J Phys Chem 87:1984-1990

43. Ghosh G, Aswal VK, Varade D (2008) A small angle neutron scattering study on the mixtures of pluronic L121 and anionic surfactant AOT. Pramana J Phys 71:1063-1067 
44. Aharoni SM (1983) On entanglements of flexible and rodlike polymers. Macromolecules 16:1722-1728

45. Fischer J, Paschek D, Geiger A, Sadowski G (2008) Modeling of aqueous poly(oxyethylene) solutions: 1. Atomistic simulations. J Phys Chem B 112:2388-2398

46. Kawaguchi S, Imai G, Suzuki J, Miyahara A, Kitano T, Ito K (1997) Aqueous solution properties of oligo- and poly(ethylene oxide) by static light scattering and intrinsic viscosity. Polymer 38:2885-2891

47. Devanand K, Selser JC (1991) Asymptotic behavior and longrange interactions in aqueous solutions of poly(ethylene oxide). Macromolecules 24:5943-5947

48. Zhang L, Eisenberg A (1996) Multiple morphologies and characteristics of "Crew-Cut" micelle-like aggregates of polystyrene-b-poly(acrylic acid) diblock copolymers in aqueous solutions. J Am Chem Soc 118:3168-3181

49. Varade D, Rodríguez C, Shrestha LK, Aramaki K (2007) Wormlike micelles in mixed surfactant systems: effect of cosolvents. J Phys Chem B 111:10438-10447

50. Ganguly R, Aswal VK, Hassan PA, Gopalakrishnan IK, Kulshreshtha SK (2006) Effect of SDS on the self-assembly behavior of the PEO-PPO-PEO triblock copolymer $(\mathrm{EO})_{20}(\mathrm{PO})_{70}(\mathrm{EO})_{20}$. J Phys Chem B 110:9843-9849

51. Sheu EY, Chen SH, Huang JS (1987) Structure and growth of bis (2-ethylhexyl) sulfosuccinate micelles in aqueous solutions. J Phys Chem 91:3306-3310

52. Bryskhe K, Jansson J, Topgaard D, Schillén K, Olsson U (2004) Spontaneous vesicle formation in a block copolymer system. J Phys Chem B 108:9710-9719

53. Fan Y, Cao M, Yuan G, Wang Y, Yan H, Ha CC (2006) Aggregation behavior in mixed system of double-chained anionic surfactant with single-chained nonionic surfactant in aqueous solution. J Colloid Interface Sci 299:928-937

54. Israelachvili JN (1992) Intermolecular and surface forces. Academic Press, London

55. Couderc S, Li Y, Bloor DM, Holzwarth JF, Wyn-Jones E (2001) Interaction between the nonionic surfactant hexaethylene glycol mono-n-dodecyl ether $\left(\mathrm{C}_{12} \mathrm{EO}_{6}\right)$ and the surface active nonionic ABA block copolymer Pluronic F127 ( $\left.\mathrm{EO}_{97} \mathrm{PO}_{69} \mathrm{EO}_{97}\right)$-formation of mixed micelles studied using isothermal titration calorimetry and differential scanning calorimetry. Langmuir 17:4818-4824

56. Cardoso da Silva R, Olofsson G, Schillen K, Loh W (2002) Influence of ionic surfactants on the aggregation of Poly(Ethylene Oxide)-Poly(Propylene Oxide)-Poly(Ethylene Oxide) block copolymers studied by differential scanning and isothermal titration calorimetry. J Phys Chem B 106:1239-1246

57. Löf D, Niemiec A, Schillén K, Loh W, Olofsson G (2007) A calorimetry and light scattering study of the formation and shape transition of mixed micelles of $\mathrm{EO}_{20} \mathrm{PO}_{68} \mathrm{EO}_{20}$ triblock copolymer (P123) and nonionic surfactant $\left(\mathrm{C}_{12} \mathrm{EO}_{6}\right)$. J Phys Chem $\mathrm{B}$ 111:5911-5920

58. Šarac B, Bešter-Rogač M (2009) Temperature dependent and salt inducet micellization of dodecyltrimethylammonium chloride in aqueous solution: a thermodynamic study. J Colloid Interface Sci 338:216-221

59. Phillips JN (1955) The energetics of micelle formation. J Trans Soc 51:561-568

60. Bouchemal K, Agnely F, Koffi A, Ponchel G (2009) A concise analsis of the effect of temperature and propandiol-1, 2 on Pluronic F127 micellization using isothermyl titration microcalorimetry. J Colloid Interface Sci 338:169-176

61. Hua XY, Rosen MJ (1988) Dynamic surface tension of aqueous surfactant solutions: I. Basic paremeters. J Colloid Interface Sci 124:652-659

62. Zhmud BV, Tiberg F, Kizlling J (2000) Dynamic surface tension in concentrated solutions of CnEm surfactants: a comparison between the theory and experiment. Langmuir 16:2557-2265

63. Shrestha LK, Matsumoto Y, Ihara K, Aramaki K (2008) Dynamic surface tension and surface dilatational elasticity properties of mixed surfactant/protein systems. J Oleo Sci 57:485-494

64. Rodríguez-Abreu C, Kunieda, H (2005) Equilibrium and dynamic surface tension properties of aqueous solutions of sulfonated cationicnonionic fluorocarbon surfactants. J Disper Sci Technol 26:435-440

65. Ekwall P, Mandell L, Fontell K (1970) Some observations on binary and ternary aerosol OT systems. J Colloid Interface Sci 33:215-235

66. Nagarajan R (2001) Solubilization of "guest" molecules into polymeric aggregates. Polym Adv Technol 12:23-43 